\title{
Prevalência e fatores associados ao consumo de frutas, legumes e verduras entre adolescentes de escolas públicas de Caruaru, PE
}

\author{
Prevalence and factors associated with the consumption of fruit \\ and vegetables by adolescents in public schools in Caruaru, \\ Pernambuco state
}

Ludmila Correa Muniz ${ }^{1}$

Roberta de Vargas Zanini ${ }^{1}$

Bruna Celestino Schneider ${ }^{1}$

Rafael Miranda Tassitano ${ }^{2}$

Wallacy Milton do Nascimento Feitosa ${ }^{3}$

David Alejandro González-Chica ${ }^{4}$

${ }^{1}$ Programa de

Pós-Graduação em Epidemiologia,

Universidade Federal de

Pelotas. Rua Marechal

Deodoro $1160 / 3^{\circ}$.

96020-22 Pelotas RS.

ludmuniz@yahoo.com.br

${ }^{2}$ Departamento de Educação

Física, Universidade Federal

Rural de Pernambuco.

${ }^{3}$ Associação Caruaruense de

Ensino Superior, Faculdade

ASCES.

${ }^{4}$ Programa de

Pós-Graduação em

Nutrição, Universidade

Federal de Santa Catarina.

\begin{abstract}
This is a cross-sectional school-based study that evaluated the prevalence and associated factors of the daily intake of fruit and vegetables by adolescents at public schools in Caruaru, Pernambuco. Poisson regression with a hierarchical model, including sociodemographic variables in the first hierarchical level, behavioral variables in the second and diet-related variables in the third was used to assess the factors associated with fruit and vegetable intake. The results showed that of the 600 adolescents interviewed, 10\% reported never eating fruit and $30.7 \%$ mentioned not eating vegetables. Prevalence of fruit intake was $32.9 \%$ and vegetables $29.0 \%$. Only $6.5 \%$ reported eating both kinds of food daily. Merely the daily vegetable intake showed a significant difference according to sex (higher among girls). Daily fruit intake was $63 \%$ higher among adolescents living in urban areas. Not consuming alcohol was associated with higher fruit intake, though not to vegetable intake. Daily intake of fruits and vegetables was 2.4 times higher among adolescents who consumed rice and beans daily. Daily fruit and vegetable intake among adolescents in Caruaru is below the recommendations, and was associated with gender, residence area, consumption of alcoholic beverages and consumption of rice and beans.
\end{abstract}

Key words Food consumption, Fruit, Vegetables, Adolescent
Resumo Trata-se de um estudo transversal de base escolar que avaliou a prevalência e fatores associados ao consumo diário de frutas, legumes e verduras (FLV) entre adolescentes de escolas públicas de Caruaru (PE). Para avaliar os fatores associados ao consumo diário de FLV, realizou-se análise multivariável e hierarquizada, por regressão de Poisson, com variáveis sociodemográficas no primeiro nível, comportamentais no segundo e alimentares no terceiro. Os resultados mostraram que, dos 600 adolescentes entrevistados, 10\% informaram não consumir frutas e 30,7\% não consomem legumes/verduras. A prevalência de consumo diário de frutas foi 32,9\% e de legumes/ verduras $29 \%$. Apenas $6,5 \%$ dos adolescentes consomem ambos os alimentos diariamente. Somente o consumo diário de legumes/verduras apresentou diferença segundo sexo (maior nas meninas). O consumo diário de frutas foi $63 \%$ maior entre residentes da zona urbana. Não ingerir álcool esteve associado com maior consumo de frutas, mas não com o de legumes/verduras. O consumo diário de frutas e legumes/verduras foi 2,4 vezes maior entre adolescentes que consomem arroz e feijão diariamente. O consumo diário de FLV entre adolescentes caruaruenses é inferior ao recomendado, estando associado ao sexo, zona de residência, consumo de álcool e de arroz e feijão. Palavras-chaves Consumo alimentar, Frutas, Hortaliças, Adolescente 


\section{Introdução}

De acordo com a Organização Mundial de Saúde (OMS), as doenças crônicas não transmissíveis são responsáveis por cerca de $60 \%$ do total de mortes relatadas no mundo e $46 \%$ da carga total de doenças que atinge a população mundial ${ }^{1}$. Estudos recentes sobre dieta, nutrição e prevenção de doenças crônicas apresentam fortes evidências acerca do papel da alimentação na proteção e controle de morbidades como diabetes, doenças cardiovasculares, alguns tipos de câncer, entre outras ${ }^{2}$.

O consumo insuficiente de frutas, legumes e verduras encontra-se entre os dez principais fatores de risco para a carga global de doenças em todo o mundo ${ }^{1}$. Aproximadamente 2,7 milhões de óbitos podem ser atribuídos a um baixo consumo de frutas e vegetais'. Tais alimentos são considerados componentes importantes de uma dieta saudável, pois são fontes de fibras, micronutrientes e outros componentes com propriedades funcionais ${ }^{1}$.

Apesar dessas evidências, o consumo de frutas, legumes e verduras ainda é insuficiente, tanto em países desenvolvidos quanto em países em desenvolvimento como o Brasil'2. A OMS define como adequado o consumo mínimo de 400 gramas por dia destes alimentos (equivalente a cinco porções diárias) $)^{3}$. Atualmente, apenas uma minoria da população atinge a recomendação de consumo diário de frutas e vegetais ${ }^{2}$, sobretudo indivíduos mais jovens, entre os quais o consumo é ainda menor ${ }^{4,5}$.

No Brasil, existem poucos trabalhos publicados que avaliam o consumo de frutas e vegetais entre adolescentes. Um estudo de base escolar realizado com jovens de 14 a 19 anos de idade, em Londrina (PR), encontrou uma prevalência de consumo inadequado de frutas e verduras, definido como uma ingestão inferior a quatro vezes por semana, de $56,7 \%$ e $43,9 \%$, respectivamente ${ }^{6}$. Outro estudo, realizado em Florianópolis (SC), em 2005, mostrou uma prevalência de consumo adequado de frutas, legumes e verduras, definido como consumo $>5$ vezes $/$ dia, de $21,2 \%$ entre indivíduos de 18 a 24 anos de idade $e^{4}$.

Tais achados são preocupantes, pois é durante a adolescência que os hábitos alimentares estão sendo adquiridos e consolidados. Além disso, hábitos alimentares adotados nessa fase da vida têm grande influência sobre o crescimento e desenvolvimento e, podem permanecer na idade adulta, repercutindo, muitas vezes, de maneira negativa sobre a saúde dos indivíduos ${ }^{7,8}$.
Considerando a escassez de estudos sobre este assunto, especialmente nas regiões norte e nordeste do Brasil, assim como a sua relevância para o estabelecimento de políticas públicas que visem reduzir o risco futuro de doenças crônicas, $o$ presente estudo tem por objetivo descrever a prevalência de consumo diário de frutas, legumes e verduras por adolescentes caruaruenses e avaliar os fatores associados ao seu consumo.

\section{Métodos}

Caruaru é uma cidade de médio porte ( 290 mil habitantes) localizada na região do agreste pernambucano, a $120 \mathrm{~km}$ da capital do estado, Recife. Em outubro de 2007, realizou-se um estudo transversal com alunos de 15 a 20 anos, matriculados nas escolas de ensino médio da rede pública estadual do município. À época do estudo, Caruaru, que é dividida em três regiões (norte, centro e sul), contava com 15 escolas públicas estaduais: quatro escolas de grande porte $(>500$ alunos), sete de tamanho médio (200-499 alunos) e quatro de pequeno porte ( $<200$ alunos). Estas escolas ofereciam ensino médio para 8.833 alunos, distribuídos em 215 turmas (109 diurnas e 106 noturnas). Esses dados são referentes ao censo escolar de 2007 e foram fornecidos pela Secretaria da Educação e Cultura (Seduc) do Estado de Pernambuco.

O cálculo do tamanho da amostra foi realizado com o auxílio do programa SampleXS, distribuído pela OMS para apoiar o planejamento amostral em estudos transversais. Foram utilizados os seguintes parâmetros e estimativas: população $(\mathrm{n}=8.833)$, intervalo de confiança de 95\%, erro máximo tolerável de cinco pontos percentuais, efeito de delineamento amostral de 1,5 e prevalência estimada de $50 \%$, por não se conhecer com precisão as diversas condutas de risco à saúde entre estudantes do ensino médio de Caruaru. Com base nesses parâmetros o tamanho amostral necessário foi de 520 adolescentes. Adicionalmente, foram acrescidos $20 \%$ para eventuais perdas e recusas e controle para fatores de confusão. Dessa forma, a amostra final necessária foi de 649 estudantes, sendo considerados elegíveis todos aqueles que se encontravam em sala de aula no dia da coleta dos dados.

O processo de amostragem foi realizado em dois estágios. Todos os sorteios foram realizados mediante o programa randomizer, que forneceu números aleatórios. Numa primeira etapa foram selecionadas oito escolas estaduais, que 
foram escolhidas de forma proporcional ao tamanho, garantindo que pelo menos 50\% das escolas de cada tamanho fossem selecionadas ( 2 escolas de grande porte, 4 de médio porte e 2 pequenas). Este procedimento foi adotado a fim de que a amostra mantivesse a distribuição das escolas pelo município.

Em um segundo momento foram selecionadas aleatoriamente duas turmas em cada uma das oito escolas incluídas na primeira fase (16 turmas no total). Segundo as informações fornecidas pela Seduc considerou-se que havia, em média, 41 alunos matriculados em cada turma de ensino médio das escolas estaduais do município, o que totalizaria 656 alunos nas turmas selecionadas. O número médio de alunos matriculados em cada turma foi similar independentemente do tamanho das escolas.

As informações sobre os desfechos em estudo (consumo diário de frutas, consumo diário de legumes/verduras, e consumo diário de ambos os alimentos: frutas e legumes/verduras), foram coletadas a partir de duas perguntas do bloco de questões referente ao consumo alimentar: Quantas vezes você come frutas (não incluindo suco de frutas)? e Quantas vezes você come legumes (cenoura, vagem, abóbora, couve-flor, etc.)?. As frequências de consumo foram coletadas da seguinte forma no momento da entrevista: nunca, 1 a 3 vezes por semana, 4 a 6 vezes por semana, 1 vez por dia, 2 vezes por dia e 3 ou mais vezes por dia. No presente estudo foram considerados positivos para os desfechos aqueles adolescentes que referiram uma frequência de consumo igual ou superior a uma vez por dia.

Foram avaliadas as seguintes variáveis independentes: sexo (masculino/feminino); idade (1517/18-20 anos); densidade do domicílio (1-4 ou $>5$ moradores); renda familiar (até R \$500, R\$501$1.000 \mathrm{ou}>\mathrm{R} \$ 1.001$ ); zona de residência (rural/ urbana); turno escolar (diurno/noturno); uso de álcool atual, independentemente da frequência e da quantidade ingerida ( $\operatorname{sim} /$ não); hábito de fumar atual, independentemente do número de cigarros fumados por dia ( $\operatorname{sim} /$ não); autopercepção de saúde (regular/ruim, boa ou excelente); número de refeições realizadas por dia (realizar ao menos 3 refeições/dia: nunca/raramente, às vezes ou sempre); consumo de alimentos, que não frutas e legumes, associados ao consumo de alimentos saudáveis (consumo diário de feijão com arroz) e ao consumo de alimentos não saudáveis (consumo diário de refrigerantes, salgadinhos fritos e doces); prática de atividade física (insuficientemente ativo: sim/não); e excesso de peso ( $\operatorname{sim} /$ não). As informações sobre atividade física foram obtidas a partir da versão curta do Questionário Internacional de Atividade Física (IPAQ) ${ }^{9}$. Foram considerados insuficientemente ativos os indivíduos que informaram praticar menos de 150 minutos de atividade física por semana.

A técnica para a coleta das medidas antropométricas (peso e altura) seguiu as recomendações de Lohman et al. ${ }^{10}$. A medida do peso corporal foi obtida utilizando uma balança eletrônica da marca Plenna (modelo Sport), com capacidade máxima de $150 \mathrm{Kg}$ e precisão de 100 gramas. A estatura foi aferida com o auxílio de um estadiômetro da marca Plenna (modelo 206), com altura máxima de 2,2 metros e precisão de 5 cm. Para classificação do estado nutricional dos indivíduos utilizou-se os critérios da $\mathrm{OMS}^{11,12}$, com base no índice de massa corporal (IMC) obtido pela divisão do peso (em quilogramas) pela altura (em metros) ao quadrado. Foram considerados com excesso de peso os adolescentes que apresentaram IMC/idade $>+1,0$ escores z na curva de referência da OMS e, para aqueles indivíduos com 20 anos de idade, foi usado como ponto de corte o IMC $>25 \mathrm{Kg} / \mathrm{m}^{2}$.

Para a coleta das informações, utilizou-se o questionário sobre "Comportamentos de Risco em Adolescentes Catarinenses" (Compac), previamente validado em estudantes da rede pública de ensino brasileira ${ }^{13}$. O questionário abrangia informações sociodemográficas e sobre trabalho, além de cinco sessões de questões relacionadas à saúde (hábitos alimentares, atividade física, comportamentos de risco, comportamentos preventivos e percepção da saúde).

O questionário foi previamente testado através de um estudo piloto, realizado com 60 adolescentes de 15 a 20 anos de idade de duas escolas caruaruenses não selecionadas para participar do estudo. Previamente à aplicação do instrumento, os estudantes foram informados sobre os objetivos da pesquisa e sobre a preservação do anonimato, sendo que os mesmos somente poderiam participar do estudo mediante a apresentação do termo de consentimento esclarecido assinado pelos pais ou responsáveis. Aceitou-se a assinatura do próprio estudante no caso do mesmo ter 18 ou mais anos de idade. O questionário foi aplicado em sala de aula (entrevista coletiva/autoaplicada) por equipe treinada e sob a supervisão dos pesquisadores responsáveis pelo estudo. O tempo médio para a aplicação do questionário foi de 30 a 40 minutos.

Foi utilizado o programa Epi Data (versão 3.1) para a digitação dos questionários. A análise 
estatística foi realizada com programa Stata (versão 11.0). O efeito de amostragem por conglomerados foi considerado em todas as análises, utilizando-se o conjunto de comandos survey (svy) do Stata 11.0. Para a análise dos dados utilizou-se um modelo hierárquico para a determinação do desfecho (Figura 1), no qual as variáveis sociodemográficas situavam-se no primeiro nível (mais distal), as comportamentais no segundo nível (intermediário) e aquelas relacionadas ao consumo alimentar no nível mais proximal ao desfecho. A significância estatística foi avaliada através do teste de Wald, por meio de regressão de Poisson ${ }^{14}$, com nível de significância de $5 \%$. Na análise ajustada foram mantidas no modelo as variáveis com valor $\mathrm{p}<0,20$. $\mathrm{O}$ ajuste para fatores de confusão utilizou um modelo hierárquico, onde o efeito de cada variável sobre o desfecho era controlado por aquelas que estavam no mesmo nível ou acima.

O estudo foi aprovado pelo Comitê de Ética em Pesquisa com seres humanos da Associação Caruaruense de Ensino Superior (ASCES).

\section{Resultados}

Um total de oito escolas e 16 turmas participaram da pesquisa. Dentre todos os estudantes que estavam presentes nos dias de coleta $(n=624), 21$ recu-

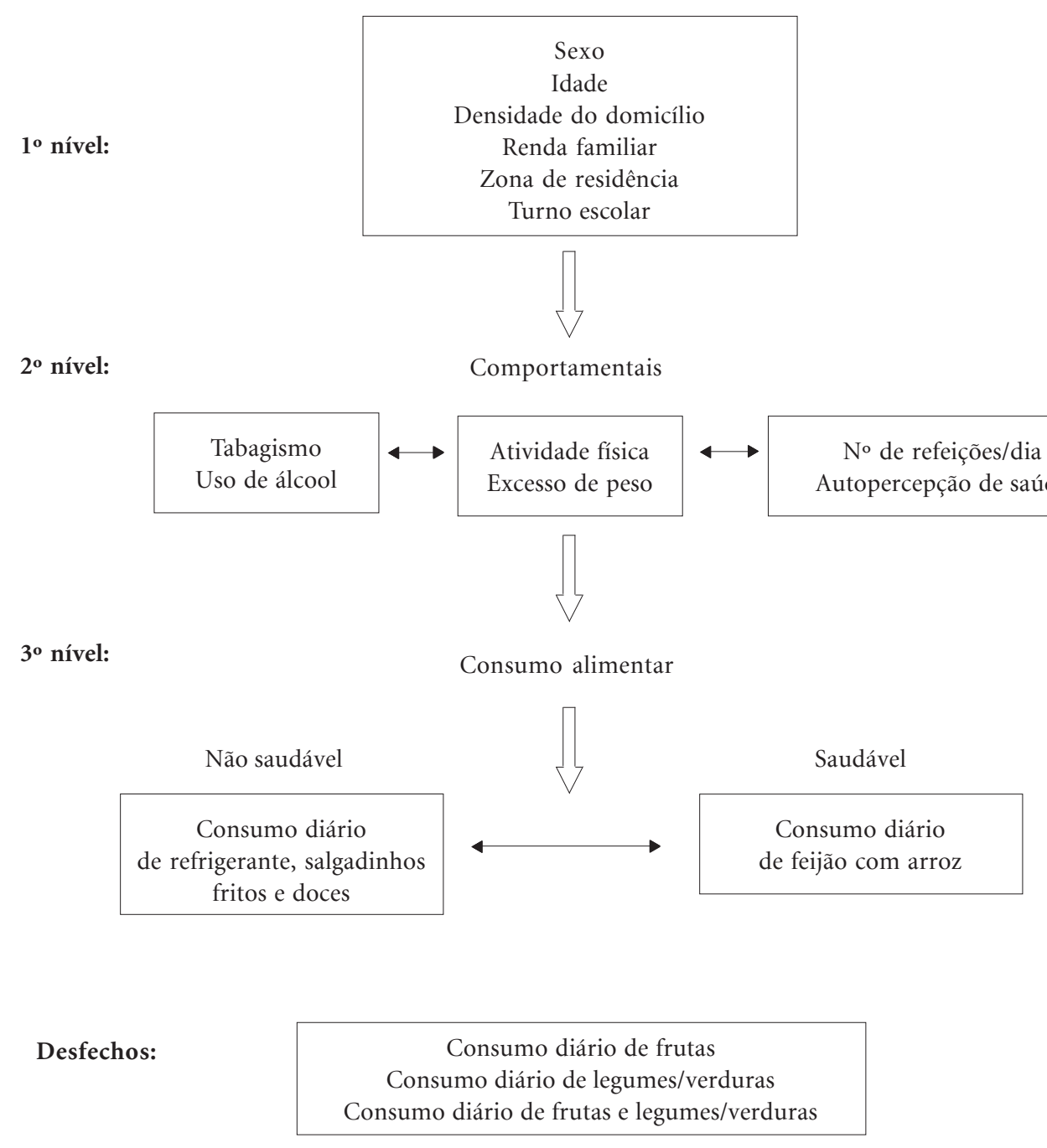

Figura 1. Modelo hierárquico, conceitual, para análise do consumo diário de frutas e/ou legumes/verduras em adolescentes de Caruaru-PE 
saram participar e em 3 casos não foram obtidas informações sobre consumo alimentar $(3,8 \%$ de perdas). A amostra final deste estudo foi composta por 600 adolescentes (62,3\% do sexo feminino), cuja média de idade foi de 17,4 anos (DP $\pm 1,5)$.

A Tabela 1 apresenta a distribuição da frequência de consumo de frutas, legumes e verduras, estratificada por sexo. Cerca de 10\% (IC95\%: $5,5 ; 14,2)$ dos adolescentes informaram nunca consumir frutas e 30,7\% (IC95\%: 25, 1; 36,3) referiram não consumir legumes/verduras. Não houve diferença estatisticamente significativa entre os sexos para o consumo de frutas e para o consumo diário de ambos os alimentos (frutas e legumes/verduras). No entanto, ao considerar os legumes/verduras, o consumo semanal (seja de 1-3 vezes ou 4-6 vezes/semana) foi maior entre as meninas em relação aos meninos.

Com relação ao consumo diário de frutas, encontrou-se uma prevalência de 32,9\% (IC95\%: $28,3 ; 37,6)$. Dentre os adolescentes que referiram consumir frutas diariamente, a maioria $(52,1 \%)$ (IC95\%: 41,0; 63,1) consome apenas uma vez ao dia. A Tabela 2 apresenta as análises bruta e ajustada entre o consumo diário de frutas e as variáveis independentes estudadas. As variáveis "zona de residência", "consumo de bebida alcoólica" e"consumo diário de frituras" foram as únicas que se mostraram associadas de forma significativa com o desfecho, mesmo após ajuste para confusão. $\mathrm{Na}$ análise ajustada a prevalência de consumo diário de frutas foi $60 \%$ maior entre os adolescentes residentes na zona urbana, em comparação aos que residem na zona rural; $30 \%$ maior entre os adolescentes que não ingerem bebidas alcoólicas, em comparação aos que fazem uso de álcool; e 15\% menor entre aqueles que não consomem frituras diariamente em relação aos que consomem.

A prevalência de consumo diário de legumes/ verduras foi de 29\% (IC95\%: 25,4; 32,6). Aproximadamente 68\% (IC95\%: 61,7; 73,6) dos adolescentes que informaram consumir legumes/verduras diariamente, o fazem somente uma vez por dia. A Tabela 3 mostra as RP brutas e ajustadas do consumo diário de legumes/verduras de acordo com as variáveis de exposição estudadas. Similar ao observado para o consumo de frutas, muitas das exposições não apresentaram associação significativa com o desfecho, mesmo após ajuste para possíveis fatores de confusão. $\mathrm{Na}$ análise ajustada a prevalência de consumo diário de legumes/verduras foi $21 \%$ maior entre as meninas, em comparação aos meninos.

A Tabela 4 apresenta os resultados referentes ao consumo diário de frutas e legumes/verduras. A prevalência de consumo diário destes alimentos foi de 6,5\% (IC95\%: 4,8; 8,3). A variável "consumo diário de arroz e feijão" foi a única que esteve associada com o desfecho, mesmo após ajuste para confusão.

Tabela 1. Distribuição da população estudada em termos de frequência de consumo de frutas e legumes/ verduras, segundo sexo. Caruaru-PE, 2007.

\begin{tabular}{|c|c|c|c|c|c|c|c|}
\hline \multirow[b]{2}{*}{ Frequência de Consumo } & \multicolumn{2}{|c|}{ Todos $(n=600)$} & \multicolumn{2}{|c|}{ Meninos $(n=226)$} & \multicolumn{2}{|c|}{ Meninas $(\mathrm{n}=374)$} & \multirow[b]{2}{*}{$p^{a}$} \\
\hline & $\%$ & $\mathrm{IC}_{95 \%}$ & $\%$ & $\mathrm{IC}_{95 \%}$ & $\%$ & $\mathrm{IC}_{95 \%}$ & \\
\hline Frutas & & & & & & & 0,4 \\
\hline Nunca & 9,8 & $5,5-14,2$ & 9,5 & $4,2-14,8$ & 10,1 & $4,3-15,8$ & \\
\hline $1-3$ vezes/semana & 40,2 & $36,0-44,5$ & 39,1 & $35,3-43,0$ & 40,8 & $34,6-47,1$ & \\
\hline 4-6 vezes/semana & 17,0 & $14,3-19,6$ & 20,3 & $14,7-25,9$ & 15,0 & $12,3-17,7$ & \\
\hline $1-2$ vezes/dia & 26,0 & $22,2-29,8$ & 23,9 & $18,3-29,4$ & 27,3 & $21,5-33,0$ & \\
\hline 3 vezes/dia & 7,0 & $5,2-8,7$ & 7,2 & $4,5-9,9$ & 6,8 & $3,0-10,6$ & \\
\hline Legumes/verduras & & & & & & & 0,01 \\
\hline Nunca & 30,7 & $25,1-36,3$ & 24,0 & $18,4-29,5$ & 34,6 & $28,0-41,3$ & \\
\hline $1-3$ vezes/semana & 31,4 & $27,8-35,0$ & 38,2 & $32,5-44,0$ & 27,4 & $23,7-31,0$ & \\
\hline 4-6 vezes/semana & 8,9 & $6,9-10,9$ & 12,0 & $9,1-14,9$ & 7,1 & $3,9-10,1$ & \\
\hline $1-2$ vezes/dia & 24,7 & $22,1-27,4$ & 22,6 & $19,0-26,1$ & 26,0 & $22,2-29,9$ & \\
\hline 3 vezes/dia & 4,3 & $2,9-5,6$ & 3,2 & $1,2-6,3$ & 4,9 & $2,8-7,0$ & \\
\hline Frutas e legumes/verduras & & & & & & & 0,4 \\
\hline Diariamente & 6,5 & $4,8-8,3$ & 4,6 & $0,1-9,6$ & 7,7 & $5,1-10,2$ & \\
\hline
\end{tabular}

Percentual máximo de observações desconhecidas: $4 \%(\mathrm{n}=9)$ para as variáveis frequência de consumo de legumes e consumo de frutas e legumes entre os meninos. ${ }^{\text {a }}$ Teste qui-quadrado de heterogeneidade. 
Tabela 2. Análise bruta e ajustada da associação entre consumo diário de frutas e variáveis independentes. Caruaru-PE, 2007. $(\mathrm{N}=600)$.

\begin{tabular}{|c|c|c|c|c|c|c|}
\hline Variável & $\mathbf{N}$ & Prevalência & RP bruta $\left(\mathrm{IC}_{95 \%}\right)$ & $p^{\mathrm{a}}$ & $R P$ ajustada* $\left(\mathrm{IC}_{95 \%}\right)$ & $p^{\mathrm{a}}$ \\
\hline \multicolumn{7}{|l|}{ Sexo } \\
\hline Masculino & 222 & 31,1 & 1,00 & \multirow[t]{2}{*}{0,6} & 1,00 & \multirow[t]{2}{*}{0,6} \\
\hline Feminino & 367 & 34,1 & $1,10(0,72-1,67)$ & & $1,11(0,70-1,75)$ & \\
\hline \multicolumn{7}{|l|}{ Idade (anos) } \\
\hline $15-17$ & 327 & 33,9 & 1,00 & \multirow[t]{2}{*}{0,6} & 1,00 & \multirow[t]{2}{*}{0,5} \\
\hline $18-20$ & 262 & 31,7 & $0,93(0,70-1,24)$ & & $0,92(0,69-1,22)$ & \\
\hline \multicolumn{7}{|l|}{ Número de moradores } \\
\hline$\geq 5$ & 213 & 33,4 & 1,00 & \multirow[t]{2}{*}{0,8} & 1,00 & \multirow[t]{2}{*}{0,8} \\
\hline $1-4$ & 326 & 31,9 & $1,05(0,71-1,54)$ & & $1,06(0,69-1,63)$ & \\
\hline \multicolumn{7}{|l|}{ Renda Familiar (R\$) } \\
\hline Até 500 & 246 & 34,2 & 1,00 & \multirow[t]{3}{*}{$0,5^{\mathrm{b}}$} & 1,00 & \multirow[t]{3}{*}{$0,4^{\mathrm{b}}$} \\
\hline $501-1.000$ & 185 & 32,4 & $0,95(0,78-1,15)$ & & $0,94(0,78-1,12)$ & \\
\hline$\geq 1.001$ & 158 & 31,7 & $0,93(0,70-1,23)$ & & $0,90(0,70-1,16)$ & \\
\hline \multicolumn{7}{|l|}{ Zona de residência } \\
\hline Rural & 70 & 21,4 & 1,00 & \multirow[t]{2}{*}{0,04} & 1,00 & \multirow[t]{2}{*}{0,04} \\
\hline Urbana & 519 & 34,5 & $1,61(1,01-2,57)$ & & $1,63(1,03-2,57)$ & \\
\hline \multicolumn{7}{|l|}{ Turno escolar } \\
\hline Noturno & 323 & 33,8 & 1,00 & \multirow[t]{2}{*}{0,6} & 1,00 & \multirow[t]{2}{*}{0,7} \\
\hline Diurno & 266 & 32,0 & $0,95(0,73-1,22)$ & & $0,96(0,72-1,27)$ & \\
\hline \multicolumn{7}{|l|}{ Tabagismo } \\
\hline Sim & 21 & 42,9 & 1,00 & \multirow[t]{2}{*}{0,02} & 1,00 & 0,05 \\
\hline Não & 568 & 32,6 & $0,76(0,61-0,94)$ & & $0,73(0,53-1,00)$ & \\
\hline Consumo de bebida a & & & & & & \\
\hline Sim & 274 & 28,8 & 1,00 & 0,09 & 1,00 & 0,04 \\
\hline Não & 315 & 36,5 & $1,27(0,95-1,68)$ & & $1,29(1,02-1,64)$ & \\
\hline Insuficientemente ati & & & & & & \\
\hline Sim & 241 & 33,2 & 1,00 & 0,9 & 1,00 & 1,0 \\
\hline Não & 341 & 33,1 & $0,99(0,68-1,47)$ & & $1,01(0,66-1,55)$ & \\
\hline Autopercepção de saú & & & & & & \\
\hline Regular/ruim & 128 & 28,1 & 1,00 & 0,07 & 1,00 & 0,09 \\
\hline Boa & 269 & 28,3 & $1,01(0,64-1,57)$ & & $0,97(0,63-1,52)$ & \\
\hline Excelente & 183 & 41,5 & $1,48(0,90-2,42)$ & & $1,41(0,89-2,24)$ & \\
\hline Excesso de peso & & & & & & \\
\hline Sim & 92 & 30,4 & 1,00 & 0,5 & 1,00 & 0,4 \\
\hline Não & 475 & 33,9 & $1,11(0,77-1,61)$ & & $1,15(0,77-1,73)$ & \\
\hline Realiza três refeições/c & & & & & & \\
\hline Nunca/raramente & 27 & 33,3 & 1,00 & 0,7 & 1,00 & 1,0 \\
\hline Âs vezes & 155 & 30,3 & $0,91 \quad(0,42-1,98)$ & & $0,95(0,46-2,00)$ & \\
\hline Sempre & 403 & 34,2 & $1,03(0,57-1,84)$ & & $0,96(0,52-1,76)$ & \\
\hline Consumo diário de re & & & & & & \\
\hline Sim & 172 & 32,0 & 1,00 & 0,7 & 1,00 & 0,2 \\
\hline Não & 400 & 33,5 & $1,05(0,83-1,32)$ & & $1,14(0,90-1,45)$ & \\
\hline Consumo diário de fri & & & & & & \\
\hline Sim & 162 & 35,2 & 1,00 & 0,3 & 1,00 & 0,01 \\
\hline Não & 411 & 32,1 & $0,91(0,75-1,12)$ & & $0,85(0,75-0,96)$ & \\
\hline Consumo diário de do & & & & & & \\
\hline Sim & 246 & 35,8 & 1,00 & 0,03 & 1,00 & 0,3 \\
\hline Não & 334 & 30,8 & $0,86(0,76-0,98)$ & & $0,90(0,72-1,11)$ & \\
\hline Consumo diário de ar & & & & & & \\
\hline Não & 101 & 29,7 & 1,00 & 0,4 & 1,00 & 0,4 \\
\hline Sim & 477 & 33,8 & $1,14(0,82-1,57)$ & & $1,13(0,81-1,61)$ & \\
\hline
\end{tabular}

Percentual máximo de observações desconhecidas: 10,2\% ( $n=61$, variável número de moradores). ${ }^{a}$ Teste de heterogeneidade (Wald). ${ }^{\mathrm{b}}$ Teste de Wald para tendência linear. 
Tabela 3. Análise bruta e ajustada da associação entre consumo diário de legumes/verduras e variáveis independentes. Caruaru-PE, 2007. $(\mathrm{N}=600)$.

\begin{tabular}{|c|c|c|c|c|c|c|}
\hline Variável & $\mathrm{N}$ & Prevalência & RP bruta $\left(\mathrm{IC}_{95 \%}\right)$ & $p^{\mathrm{a}}$ & $\mathrm{RP}$ ajustada ${ }^{\star}\left(\mathrm{IC}_{95 \%}\right)$ & $p^{\mathrm{a}}$ \\
\hline \multicolumn{7}{|l|}{ Sexo } \\
\hline Masculino & 217 & 25,8 & 1,00 & \multirow[t]{2}{*}{0,006} & 1,00 & \multirow[t]{2}{*}{0,04} \\
\hline Feminino & 369 & 30,9 & $1,20(1,06-1,34)$ & & $1,21(1,01-1,45)$ & \\
\hline \multicolumn{7}{|l|}{ Idade (anos) } \\
\hline $15-17$ & 324 & 26,9 & 1,00 & \multirow[t]{2}{*}{0,1} & 1,00 & \multirow[t]{2}{*}{0,2} \\
\hline $18-20$ & 262 & 31,7 & $1,18(0,94-1,48)$ & & $1,17(0,89-1,55)$ & \\
\hline \multicolumn{7}{|l|}{ Número de moradores } \\
\hline$\geq 5$ & 212 & 25,9 & 1,00 & \multirow[t]{2}{*}{0,05} & 1,00 & \multirow[t]{2}{*}{0,05} \\
\hline $1-4$ & 324 & 30,9 & $1,19(1,00-1,42)$ & & $1,19(1,00-1,43)$ & \\
\hline \multicolumn{7}{|l|}{ Renda Familiar (R\$) } \\
\hline Até 500 & 245 & 31,0 & 1,00 & \multirow[t]{3}{*}{$0,09^{\mathrm{b}}$} & 1,00 & \multirow[t]{3}{*}{$0,3^{\mathrm{b}}$} \\
\hline $501-1.000$ & 184 & 28,8 & $0,93(0,77-1,12)$ & & $0,94(0,73-1,20)$ & \\
\hline$\geq 1.001$ & 157 & 26,1 & $0,84(0,69-1,03)$ & & $0,90(0,72-1,12)$ & \\
\hline \multicolumn{7}{|l|}{ Zona de residência } \\
\hline Rural & 68 & 38,2 & 1,00 & \multirow[t]{2}{*}{0,1} & 1,00 & \multirow[t]{2}{*}{0,1} \\
\hline Urbana & 518 & 27,8 & $0,73(0,49-1,09)$ & & $0,73(0,50-1,07)$ & \\
\hline \multicolumn{7}{|l|}{ Turno escolar } \\
\hline Noturno & 322 & 27,6 & 1,00 & \multirow[t]{2}{*}{0,4} & 1,00 & \multirow[t]{2}{*}{0,6} \\
\hline Diurno & 264 & 30,7 & $1,11(0,86-1,43)$ & & $1,06(0,86-1,29)$ & \\
\hline \multicolumn{7}{|l|}{ Tabagismo } \\
\hline Sim & 21 & 23,8 & 1,00 & \multirow[t]{2}{*}{0,7} & 1,00 & 0,8 \\
\hline Não & 565 & 29,2 & $1,23(0,44-3,39)$ & & $1,13(0,47-2,69)$ & \\
\hline Consumo de bebida a & & & & & & \\
\hline Sim & 273 & 28,2 & 1,00 & 0,7 & 1,00 & 0,6 \\
\hline Não & 313 & 29,7 & $1,05(0,77-1,44)$ & & $0,94(0,71-1,25)$ & \\
\hline Insuficientemente ati & & & & & & \\
\hline Sim & 241 & 27,4 & 1,00 & 0,4 & 1,00 & 0,4 \\
\hline Não & 339 & 30,4 & $1,11(0,87-1,42)$ & & $1,15(0,80-1,65)$ & \\
\hline Autopercepção de saú & & & & & & \\
\hline Regular/ruim & 126 & 36,5 & 1,00 & 0,007 & 1,00 & 0,05 \\
\hline Boa & 268 & 25,4 & $0,70(0,56-0,86)$ & & $0,72(0,54-0,96)$ & \\
\hline Excelente & 183 & 28,4 & $0,78(0,49-1,25)$ & & $0,78(0,44-1,39)$ & \\
\hline Excesso de peso & & & & & & \\
\hline Sim & 90 & 30,0 & 1,00 & 0,8 & 1,00 & 0,7 \\
\hline Não & 474 & 28,3 & $0,94(0,52-1,69)$ & & $0,91(0,53-1,55)$ & \\
\hline Realiza três refeições/c & & & & & & \\
\hline Nunca/raramente & 27 & 22,2 & 1,00 & 0,6 & 1,00 & 0,4 \\
\hline Às vezes & 156 & 28,9 & $1,30(0,56-3,02)$ & & $1,53(0,54-4,36)$ & \\
\hline Sempre & 399 & 29,6 & $1,33(0,69-2,55)$ & & $1,64(0,71-3,79)$ & \\
\hline Consumo diário de re & & & & & & \\
\hline Sim & 172 & 30,8 & 1,00 & 0,4 & 1,00 & 0,6 \\
\hline Não & 397 & 28,2 & $0,92(0,73-1,15)$ & & $1,04(0,89-1,23)$ & \\
\hline Consumo diário de fri & & & & & & \\
\hline Sim & 159 & 27,0 & 1,00 & 0,4 & 1,00 & 0,8 \\
\hline Não & 411 & 30,2 & $1,12(0,84-1,50)$ & & $1,03(0,82-1,28)$ & \\
\hline Consumo diário de do & & & & & & \\
\hline Sim & 245 & 26,5 & 1,00 & 0,07 & 1,00 & 0,2 \\
\hline Não & 332 & 31,0 & $1,17(0,99-1,39)$ & & $1,12(0,93-1,35)$ & \\
\hline Consumo diário de ar & & & & & & \\
\hline Não & 102 & 25,5 & 1,00 & 0,6 & 1,00 & 0,6 \\
\hline Sim & 473 & 29,6 & $1,16(0,57-2,35)$ & & $1,20(0,55-2,64)$ & \\
\hline
\end{tabular}

Percentual máximo de observações desconhecidas: 10,7\% ( $\mathrm{n}=64$, variável número de moradores no domicílio). ${ }^{\mathrm{a}}$ Teste de

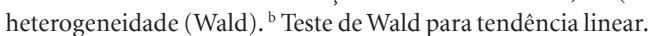


Tabela 4. Análise bruta e ajustada da associação entre consumo diário de frutas e legumes/verduras e variáveis independentes. Caruaru-PE, 2007. $(\mathrm{N}=600)$.

\begin{tabular}{|c|c|c|c|c|c|c|}
\hline Variável & $\mathbf{N}$ & Prevalência & RP bruta $\left(\mathrm{IC}_{95 \%}\right)$ & $p^{\mathrm{a}}$ & RP ajustada ${ }^{*}\left(\mathrm{IC}_{95 \%}\right)$ & $p^{\mathrm{a}}$ \\
\hline \multicolumn{7}{|l|}{ Sexo } \\
\hline Masculino & 217 & 4,6 & 1,00 & \multirow[t]{2}{*}{0,4} & 1,00 & \multirow[t]{2}{*}{0,3} \\
\hline Feminino & 366 & 7,7 & $1,66(0,48-5,76)$ & & $1,78(0,48-6,57)$ & \\
\hline \multicolumn{7}{|l|}{ Idade (anos) } \\
\hline $15-17$ & 323 & 7,1 & 1,00 & \multirow[t]{2}{*}{0,6} & 1,00 & \multirow[t]{2}{*}{0,4} \\
\hline $18-20$ & 260 & 5,8 & $0,81(0,32-2,07)$ & & $0,67(0,22-2,01)$ & \\
\hline \multicolumn{7}{|l|}{ Número de moradores } \\
\hline$\geq 5$ & 211 & 8,1 & 1,00 & \multirow[t]{2}{*}{0,1} & 1,00 & \multirow[t]{2}{*}{0,1} \\
\hline $1-4$ & 322 & 5,6 & $0,69(0,43-1,13)$ & & $0,71(0,43-1,15)$ & \\
\hline \multicolumn{7}{|l|}{ Renda Familiar (R\$) } \\
\hline Até 500 & 243 & 7,0 & 1,00 & \multirow[t]{3}{*}{0,8} & 1,00 & \multirow[t]{3}{*}{0,1} \\
\hline $501-1.000$ & 183 & 6,0 & $0,86(0,48-1,53)$ & & $0,81(0,45-1,46)$ & \\
\hline$\geq 1.001$ & 157 & 6,4 & $0,91(0,55-1,52)$ & & $0,75(0,51-1,11)$ & \\
\hline \multicolumn{7}{|l|}{ Zona de residência } \\
\hline Rural & 68 & 6,0 & 1,00 & \multirow[t]{2}{*}{0,8} & 1,00 & \multirow[t]{2}{*}{0,9} \\
\hline Urbana & 515 & 6,6 & $1,12(0,47-2,66)$ & & $1,03(0,41-2,61)$ & \\
\hline \multicolumn{7}{|l|}{ Turno escolar } \\
\hline Noturno & 319 & 7,2 & 1,00 & \multirow[t]{2}{*}{0,3} & 1,00 & \multirow[t]{2}{*}{0,4} \\
\hline Diurno & 264 & 5,7 & $0,79(0,50-1,23)$ & & $0,76(0,41-1,42)$ & \\
\hline \multicolumn{7}{|l|}{ Tabagismo } \\
\hline Sim & 21 & 14,3 & 1,00 & \multirow[t]{2}{*}{0,1} & 1,00 & 0,1 \\
\hline Não & 562 & 6,2 & $0,44(0,14-1,32)$ & & $0,39(0,12-1,29)$ & \\
\hline Consumo de bebida al & & & & & & \\
\hline Sim & 271 & 6,6 & 1,00 & 0,9 & 1,00 & 0,7 \\
\hline Não & 312 & 6,4 & $0,97(0,52-1,79)$ & & $0,86(0,38-1,90)$ & \\
\hline Insuficientemente ati & & & & & & \\
\hline Sim & 239 & 5,9 & 1,00 & 0,6 & 1,00 & 0,5 \\
\hline Não & 338 & 6,8 & $1,16(0,66-2,04)$ & & $1,29(0,61-2,73)$ & \\
\hline Autopercepção de saú & & & & & & \\
\hline Regular/ruim & 126 & 8,7 & 1,00 & 0,1 & 1,00 & 0,1 \\
\hline Boa & 267 & 4,5 & $0,51(0,29-0,93)$ & & $0,52(0,29-0,94)$ & \\
\hline Excelente & 181 & 7,2 & $0,82(0,31-2,16)$ & & $0,82(0,31-2,19)$ & \\
\hline Excesso de peso & & & & & & \\
\hline Sim & 90 & 7,8 & 1,00 & 0,6 & 1,00 & 0,7 \\
\hline Não & 471 & 6,6 & $0,85(0,45-1,58)$ & & $1,09(0,72-1,66)$ & \\
\hline Realiza três refeições/c & & & & & & \\
\hline Nunca/raramente & 27 & 7,4 & 1,00 & 0,9 & 1,00 & 0,8 \\
\hline Às vezes & 154 & 7,1 & $0,96(0,11-8,62)$ & & $1,04(0,12-9,27)$ & \\
\hline Sempre & 398 & 6,3 & $0,85(0,10-7,27)$ & & $0,81(0,08-8,14)$ & \\
\hline Consumo diário de re & & & & & & \\
\hline $\operatorname{Sim}$ & 171 & 6,4 & 1,00 & 1,0 & 1,00 & 0,6 \\
\hline Não & 395 & 6,3 & $0,98(0,36-2,72)$ & & $0,80(0,31-2,06)$ & \\
\hline Consumo diário de fri & & & & & & \\
\hline Sim & 159 & 4,4 & 1,00 & 0,3 & 1,00 & 0,3 \\
\hline Não & 408 & 7,1 & $1,61(0,60-4,34)$ & & $1,61(0,62-4,22)$ & \\
\hline Consumo diário de do & & & & & & \\
\hline Sim & 245 & 7,4 & 1,00 & 0,5 & 1,00 & 0,5 \\
\hline Não & 329 & 5,8 & $0,79(0,36-1,70)$ & & $0,70(0,24-2,02)$ & \\
\hline Consumo diário de ar & & & & & & \\
\hline Não & 101 & 3,0 & 1,00 & 0,03 & 1,00 & 0,03 \\
\hline Sim & 471 & 7,2 & $2,43(1,11-5,32)$ & & $2,40(1,09-5,27)$ & \\
\hline
\end{tabular}

Percentual máximo de observações desconhecidas: 11,2\% ( $\mathrm{n}=67$, variável número de moradores no domicílio). ${ }^{\mathrm{a}}$ Teste de heterogeneidade (Wald) 
O consumo de frutas e legumes/verduras foi 2,4 vezes maior entre os adolescentes que referiram consumir arroz e feijão diariamente em relação aos que não consomem. Apesar da maioria dos resultados não apresentar associação estatisticamente significativa com o desfecho, maiores prevalências de consumo (pelo menos $60 \%$ em relação às respectivas categorias de referência) foram observadas entre as meninas e entre adolescentes que não consomem frituras diariamente.

Todas as análises, referentes aos três desfechos, foram repetidas de forma estratificada segundo sexo, mas não foram encontradas evidências de modificação de efeito nas associações.

\section{Discussão}

Ao realizar extensa busca na literatura, foram localizados poucos trabalhos que avaliaram o consumo de frutas e/ou vegetais entre adolescentes brasileiros, especialmente na região nordeste, fato que torna relevante os resultados deste estudo.

Uma limitação a ser considerada é que o instrumento utilizado para a coleta das informações foi autoaplicado, o que poderia levar à ocorrência de erro de recordatório e viés de informação por parte dos entrevistados. É importante destacar ainda que a amostra incluiu apenas adolescentes matriculados em escolas públicas de ensino fundamental, o que limita a extrapolação dos resultados para todos os adolescentes de Caruaru. Além disso, mesmo não sendo possível avaliar os 649 adolescentes previstos no início do estudo, o número de avaliados foi superior ao estipulado no cálculo amostral (520 adolescentes), pois a estimativa previu um acréscimo de $20 \%$ para eventuais perdas. Dessa forma, seria pouco provável que o pequeno número de perdas $(3,8 \%)$ tenha afetado os resultados encontrados.

Os resultados do presente estudo mostraram que apenas um terço dos adolescentes referiu o consumo diário de frutas ou de legumes/verduras, e menos de 7\% informaram consumir ambos os alimentos diariamente. Estas estimativas são consistentes com estudos prévios que também revelaram um consumo insuficiente de frutas e vegetais entre adolescentes ${ }^{4-6}$. Entretanto, a comparação dos resultados deste estudo com os de outros trabalhos deve ser realizada com cautela, visto que as definições de consumo insuficiente e/ou adequado para esses alimentos foram diferentes. Estudo de base escolar realizado em Londrina (PR) em 2005, com adolescentes de 14 a 19 anos de idade, mostrou uma prevalência de consumo inadequado ( $<4$ dias por semana) de $56,7 \%$ para frutas e $43,9 \%$ para verduras 6 . No mesmo ano, em Florianópolis-SC, estudo de base populacional encontrou uma prevalência de $21,2 \%$ de consumo adequado de frutas, legumes ou verduras ( $>5$ vezes/dia) entre jovens de 18 a 24 anos de idade ${ }^{4}$. Jaime et al. ${ }^{5}$, a partir de inquérito telefônico realizado nas capitais brasileiras e no Distrito Federal, mostraram que, entre indivíduos com idade entre 18 e 24 anos, a prevalência de consumo regular de frutas e hortaliças ( $>$ 5 dias/semana) foi de $14,5 \%$, sendo que a prevalência de consumo adequado desses alimentos (> 5 vezes/dia) foi de apenas 3,1\%.

A baixa frequência de consumo de frutas, legumes e verduras torna-se preocupante, visto que estes alimentos são ricos em vitaminas, minerais e fibras alimentares e, portanto, deveriam fazer parte da alimentação de indivíduos de todas as faixas etárias, pois contribuem para a proteção à saúde e redução do risco de ocorrência de várias doenças crônicas ${ }^{2,15}$. O Ministério da Saúde, através do Guia Alimentar para a População Brasileira, recomenda o consumo diário de três porções de frutas e três porções de legumes e verduras ao longo das refeições ${ }^{15}$. Neste sentido, os resultados do presente estudo chamam a atenção, não apenas por sugerirem que as recomendações de consumo preconizadas pela OMS e pelo Ministério da Saúde não estão sendo atingidas, mas porque o consumo de frutas e/ou legumes/ verduras entre os adolescentes de Caruaru não ocorre diariamente na maior parte dos casos. Além disso, considerando que o consumo insuficiente de frutas, legumes e verduras é um dos principais fatores de risco para morbimortalidade entre adultos ${ }^{1}$, o baixo consumo desses alimentos na adolescência pode se tornar um hábito alimentar persistente na vida adulta ${ }^{7,8}$.

Os resultados do presente estudo mostraram que o consumo de frutas foi semelhante em ambos os sexos, mas o consumo diário de legumes/ verduras foi maior entre as meninas. Uma revisão sistemática realizada por Rasmussen et al. ${ }^{16}$ em 2006, mostrou que 27 dos 49 estudos que avaliaram o consumo de frutas e/ou vegetais entre crianças e adolescentes encontraram maior ingestão destes alimentos entre as meninas. No entanto, os autores mencionam que a falta de consistência dos estudos quanto às diferenças no consumo de frutas e/ou vegetais entre os sexos estaria relacionada com as áreas geográficas onde os mesmos foram realizados.

A mesma revisão ${ }^{16}$ destaca também que o consumo de frutas e vegetais ocorre com maior 
frequência e em maior quantidade entre crianças e adolescentes residentes na zona rural do que na zona urbana. Estes achados são inconsistentes com os resultados do presente estudo, que mostrou maior consumo de frutas entre os adolescentes residentes na zona urbana, em comparação aos que residem na zona rural. É provável que as adversidades climáticas existentes no nordeste do Brasil ${ }^{17}$ junto com as limitações de acesso sejam responsáveis por este achado.

Embora as adversidades socioeconômicas (especialmente a menor renda familiar) estejam associadas com baixas prevalências de consumo de frutas e vegetais ${ }^{16}$, no presente estudo a renda familiar não se mostrou associada com nenhum dos desfechos. Apenas o menor número de moradores mostrou um efeito benéfico, embora pequeno, sobre o consumo de legumes/verduras. Mesmo que a renda familiar não tenha se mostrado associada aos desfechos, este achado sugere que o baixo consumo destes alimentos é um hábito não saudável que afeta de forma similar adolescentes de todos os estratos sociais. Por este motivo seriam necessárias políticas públicas mais abrangentes, as quais devem estimular o consumo de frutas, legumes e vegetais independentemente da posição socioeconômica.

Estudos recentes mostraram que alimentos ricos em açúcares e gorduras estão inversamente relacionados ao consumo de frutas e vegetais ${ }^{18,19}$. Tal comportamento poderia explicar o aumento observado no consumo de refrigerantes, pratos prontos e frituras, assim como nas prevalências de excesso de peso e obesidade entre adultos, crianças e, sobretudo, entre adolescentes, conforme apontado no relatório da Pesquisa de Orçamento Familiar (POF) de 2008-2009, inclusive nas regiões norte e nordeste do $\mathrm{Brasil}^{20}$. A relação inversa entre o consumo de alimentos saudáveis e não saudáveis, está bem estabelecida na literatura $^{18}$. Este fenômeno é semelhante ao encontrado no presente estudo, onde um maior consumo de frutas foi observado entre adolescentes que não consomem frituras. Da mesma forma, um maior consumo de frutas foi observado entre jovens que referiram não ingerir bebidas alcoólicas, enquanto que um maior consumo de frutas e legumes/verduras foi encontrado entre aqueles que consomem arroz e feijão diariamente, sendo esta última associação bastante forte em comparação com os outros resultados. Esses achados sugerem que comportamentos que caracterizam um estilo de vida saudável seriam praticados de forma conjunta na modalidade de clusters.
A falta de associação entre o consumo de frutas e/ou legumes/verduras com o estado nutricional poderia ser consequência da menor prevalência de excesso de peso no nordeste do Brasil em relação a outras regiões. Documento recente da POF mostrou uma tendência de incremento na prevalência de excesso de peso e obesidade para a população de adolescentes de todas as regiões brasileiras no período de 1974 a $2009^{20}$, sendo que entre meninas da região nordeste a prevalência passou de 5,5\% em 1974 para 17,1\% em 2009, e entre os meninos, mudou de $2,2 \%$ para $16,1 \%$, no mesmo período. As prevalências de excesso de peso referidas na POF de 2009 - que usou o mesmo critério utilizado nesta pesquisa - são similares às encontradas no presente estudo (16,2\%), mas são inferiores às encontradas nas regiões sul, sudeste e centro-oeste, que oscilam entre $20-25 \%$. Cabe destacar que, durante este período, houve também aumento na disponibilidade domiciliar de refrigerantes, gorduras e alimentos prontos, enquanto a baixa participação de frutas, legumes e verduras na dieta permaneceu relativamente constante (entre 3\% e 4\%) para as áreas metropolitanas do País, o que também poderia explicar os resultados ${ }^{21}$. No entanto, temos que considerar que por ser um estudo transversal, esta falta de associação pode estar sujeita ao viés da causalidade reversa. Este, poderia ser responsável pela falta de associação com a maioria das variáveis comportamentais e de consumo, assim como pela associação com o tabagismo, que mesmo sendo limítrofe, mostrou um maior consumo de frutas entre adolescentes que fumavam.

\section{Conclusão}

Com base nos resultados observou-se que a prevalência de consumo de frutas, legumes e verduras entre os adolescentes da rede pública de ensino do município de Caruaru-PE está aquém das recomendações atuais. Frente a isso, o papel de melhorar a alimentação dos adolescentes não deve ser encarado como um esforço individual, pois depende também de iniciativas governamentais que potencializem e impulsionem mudanças de múltiplos comportamentos visando à promoção da saúde. Investir em programas públicos, como o Programa Nacional de Alimentação Escolar, com o objetivo de facilitar o acesso a frutas e vegetais para todos os estratos sociais, seria, talvez, a medida mais ágil e eficaz para promover um maior consumo destes alimentos entre os jovens. 


\section{Colaboradores}

LC Muniz, BC Schneider e RV Zanini realizaram a análise dos dados e a escrita do manuscrito. DA González-Chica orientou todas as etapas do trabalho e participou na análise e redação do manuscrito. RM Tassitano e WMN Feitosa supervisionaram a coleta de dados e revisaram a versão final do manuscrito.

\section{Referências}

1. Organização Pan-Americana da Saúde (OPAS). Doenças crônico degenerativas e obesidade: Estratégia mundial sobre alimentação saudável, atividade física e saúde. Brasília: OPAS; 2003.

2. World Health Organization (WHO). Diet, nutrition and the prevention of chronic diseases. Report of a Joint FAO/WHO Consultation. Geneva: WHO; 2003. [acessado 2011 set 16]. Disponível em: http:// whqlibdoc.who.int/trs/WHO_TRS_916.pdf

3. Food and Agriculture Organization of the United Nations (FAO).World Health Organization (WHO). Fruit and vegetables for health: Report of a Joint FAO/WHO Workshop. Kobe, Japan; 2004. [acessado 2011 set 16]. Disponível em: http://www.fao.org/ ag/magazine/FAO-WHO-FV.pdf

4. Campos VC, Bastos JL, Gauche H, Boing AF, Assis MAA. Fatores associados ao consumo adequado de frutas, legumes e verduras em adultos de Florianópolis. Rev Bras Epidemiol 2010; 13(2):352-362.

5. Jaime PC, Figueiredo ICR, Moura EC, Malta DC. Fatores associados ao consumo de frutas e hortaliças no Brasil, 2006. Rev Saude Publica 2009; 43(Supl. 2):57-64.

6. Romanzini M, Reichert FF, Lopes AS, Petroski EL, Júnior JCF. Prevalência de fatores de risco cardiovascular em adolescentes. Cad Saude Publica 2008; 24(11):2573-2581.

7. Mikkila V, Rasanen L, Raitakari OT, Pietinen P, Viikari J. Longitudinal changes in diet from childhood into adulthood with respect to risk of cardiovascular diseases: The cardiovascular Risk in Young Finns Study. Eur J Clin Nutr 2004; 58(7):1038-1045.

8. Ness AR, Maynard M, Frankel S, Smith GD, Frobisher C, Leary SD, Emmett PM, Gunnell D. Diet in childhood and adult cardiovascular and cause mortality: the Boyd Orr cohort. Heart 2005; 91(7):894898.

9. Craig CL, Marshall AL, Sjöström M, Bauman AE, Booth ML, BE Ainsworth, Pratt M, Ekelund U, Yngve A, Sallis JF, Oja P. International physical activity questionnaire: 12-Country reliability and validity. Med Sci Sports Exerc 2003; 35(8):1381-1395.

10. Lohman TG, Roche AF, Martolell R. Anthropometric standardization reference manual. Illinois: $\mathrm{Hu}-$ man Kinetics Books; 1988.

11. World Health Organization. Global database on body mass index. [acessado 2011 set 16]. Disponível em: http://apps.who.int/bmi/index.jsp

12. World Health Organization (WHO). Multicentre Growth Reference Study Group. WHO child growth standards: length/height-for-age, weight-for-age, weight-for-length, weight-for-height and body mass index-for-age: methods and development. Geneva: WHO; 2006

13. Nahas MV, Barros MVG, Bem MFLD, Oliveira ESA, Loch MR. Estilo de vida e indicadores de saúde dos jovens catarinenses. Relatório da pesquisa desenvolvida pelo Núcleo de Pesquisa em Atividade Física e Saúde com amostra representativa dos estudantes de 15 a 19 anos matriculados nas escolas estaduais do ensino médio em Santa Catarina. Florianópolis: UFSC; 2005. 
14. Barros AJ, Hirakata VN. Alternatives for logistic regression in cross-sectional studies: an empirical comparison of models that directly estimate the prevalence ratio. BMC Med Res Methodol 2003; 3(1):22.

15. Brasil. Ministério da Saúde (MS). Secretaria de Atenção à Saúde. Coordenação Geral da Política de Alimentação e Nutrição. Guia Alimentar para a população brasileira: promovendo a alimentação saudável. Brasília: MS; 2006.

16. Rasmussen M, Krolner R, Klepp K-I, Lytle L, Brug J, Bere E, Due P. Determinants of fruit and vegetable consumption among children and adolescents: a review of the literature. Part I: quantitative studies. Int J Behav Nutr Phys Act 2006; (3):22.

17. Empresa Brasileira de Pesquisa Agropecuária (Embrapa). Aquecimento Global e Cenários Futuros da Agricultura Brasileira. Embrapa, 2008. [acessado 2011 maio 14]. Disponível em: http://www.embrapa.br/ publicacoes/tecnico/aquecimentoglobal.pdf.

18. Figueiredo ICR, Jaime PC, Monteiro CA. Fatores associados ao consumo de frutas, legumes e verduras em adultos da cidade de São Paulo. Rev Saude Publica 2008; 42(5):777-785.

19. Forshee RA, Storey ML. The role of added sugars in the diet quality of children and adolescents. $J$ AM College Nutr 2001; 20(1):32-43.

20. Instituto Brasileiro de Geografia e Estatística (IBGE). Pesquisa de Orçamentos Familiares 2008-2009. Antropometria e Estado Nutricional de Crianças, Adolescentes e Adultos no Brasil. Rio de Janeiro, 2010. [acessado 2010 out 19]. Disponível em: http://www. ibge.gov.br/home/estatistica/populacao/condicaodevida/pof/2008_2009_encaa/default.shtm

21. Levy-Costa RB, Sichieri R, Pontes NS, Monteiro CA. Disponibilidade domiciliar de alimentos no Brasil: distribuição e evolução (1974-2003). Rev Saude Publica 2005; 39(4):530-540.

Artigo apresentado em 21/05/2011

Aprovado em 10/06/2011

Versão final aprovada em 07/09/2011 